\title{
Management of Pneumomediastinum Associated with H1N1 Pneumonia: A Case Report
}

\author{
Bhavna P Singh1* ${ }^{*}$, Gurucharan S Shetty², Padmakumar Arayamparambil Vijayan ${ }^{1}$, Ullas \\ Gopalakrishna ${ }^{1}$, Garud Chandan ${ }^{1}$, Ario Santini ${ }^{3}$, Vivek Padegal ${ }^{4}$ \\ ${ }^{1}$ Fortis Hospitals, Department of Critical Care Medicine, Bangalore, India \\ 2 Fortis Hospitals, Department of Radiology, Bangalore, India \\ 3 University of Medicine, Pharmacy, Sciences and Technology of Târgu Mureş, Romania; Hon Fellow, University of \\ Edinburgh, United Kingdom \\ 4 Fortis Hospitals, Department of Pulmonology, Bangalore, India
}

\begin{abstract}
H1N1 is seen in tropical countries like India, occurring irrespective of the season. Complications of the disease are frequently encountered and there is little in the way or guidelines as to the how these should be managed. The treatment of one such complication, a recurrent pneumiomediastinum is the subject of the current paper. The management followed guidance for the treatment of a similar condition known as primary spontaneous pneumomediastinum, an uncommon condition resulting from alveolar rupture-otherwise known as the Macklin phenomenon.
\end{abstract}

Keywords: pandemic H1N1 virus, pneumomediastinum, Macklin phenomenon

Received: 27 May 2018 / Accepted: 10 December 2018

\section{INTRODUCTION}

The pandemic H1N1 or swine flu pneumonia virus is an influenza virus not previously been recognised as an infective agent prior to 2009. DNA analyses of the virus indicate that its roots are in animal influenza viruses and it is unrelated to the human seasonal $\mathrm{H} 1 \mathrm{~N} 1$ viruses that have been in general circulation since 1977.

Antibodies to the seasonal H1N1 virus do not protect against the pandemic $\mathrm{H} 1 \mathrm{~N} 1$ virus, though some studies have shown that a percentage of people age 65 and older have some immunity against the pandemic variant.

Following occurrences in 2009 in North America, the virus spread quickly worldwide and by the time WHO pronounced it to be pandemic, laboratory validated cases were reported in seventy-four countries. Since then, most countries in the world have substantiated infections caused by this virus.

The aim of the current paper is to report a successfully managed case caused by the H1N1 pandemic
2009 strain in the hope that it will aid in the guidance to physicians treating $\mathrm{H} 1 \mathrm{~N} 1$ and also promote further studies.

\section{CASE REPORT}

A 45 year old Indian male, height: $168 \mathrm{~cm}$, weight: 78 kilos; Race: Asian, occupation fabric businessman, residing in Kolar, a suburb of Bangalore City, had a history of fever, cough and breathlessness.

Prior to admission to the tertiary care unit (TCU) he had received treatment in a privately owned small hospital. A diagnosis of community acquired pneumonia had been made and he was admitted to their intensive care unit (ICU). He received piperacillin and tazobactam combination (Baxter, Illinois, USA), $4.5 \mathrm{~g}$ intravenously every 6 hours and continued for 14 days, plus azithromycin (CiplaInc, Mumbai, India) $500 \mathrm{mgv}$ once a day and continued for 5 days, and oseltamivir (Roche Holding AG Inc, Basel, Switzerland) $75 \mathrm{mg}$, oral every 12 hours, continued for 10 days. Additionally, oxygen therapy and intermittent non-invasive respira- 
tory support (NIV) was administered using a Drager Savina 300 NIV machine (Drager International, Geneva Swiss) with a setting of $12 / 6 \mathrm{~cm}$ of water continuously for fifteen days. His condition did not improve and he was referred to the Fortis Hospitals, a tertiary care hospital (TCH) in Bangalore City because of their excellent ICU and pulmonology team. According to local protocol, since it was in intra-ICU referral, he was directly admitted to ICU in the TCU of TCH.

On admittance to the TCU, an initial examination indicated that he had extensive subcutaneous emphysema with bilateral crepitus. This diagnosis was not included in the referral letter from the private hospital and it may be assumed that such a diagnosis had not been previously made. The referral letter did state that he had had a contrast enhanced computed tomography (CECT) following a non-resolving pneumonia, the films of which had been enclosed with the letter. This indicated severe right to left pneumomediastinum with extensive subcutaneous emphysema accompanied by interstitial pneumonitis features (Fig. 1A). Initially, treatment for pneumonia was continued and throat and nasal swabs sent for laboratory examination. Both throat and nasal swab tested positive for the H1N1 pandemic 2009 strain.

Non-invasive respiratory support was continued. A plain computed tomography was undertaken which showed a residual and resolving pneumomediastinum and extensive interstitial pneumonitis. Clinically, the subcutaneous emphysema had reduced dramatically.

The day after admission, the patient had a bout of coughing following which he complained of sudden onset of severe retrosternal chest pain and breathlessness. There was extensive subcutaneous emphysema spanning his whole upper body, extending into his arms. His blood oxygen saturation dropped to $70 \%$ and he went into respiratory distress.

The patient was intubated and the following drugs were prescribed. Fentanyl (Diprivan, ICI Healthcare Ltd) $50 \mathrm{microgram} /$ hour by injection plus propofol (Diprivan, ICI Healthcare Ltd) $50 \mathrm{microgram} / \mathrm{kg} / \mathrm{min}$ by injection and atracurium (Neon Lab Ltd, India) $7 \mathrm{mg} /$ hour by intravenous injection.

Sedation and paralysis were monitored for twenty-four hours during which his condition improved, and after which it was assumed that he was ready to undergo the gradual process of decreased ventilator support (weaning) since he was stable with a blood oxygen saturations $>94 \%$. To assess if he was able

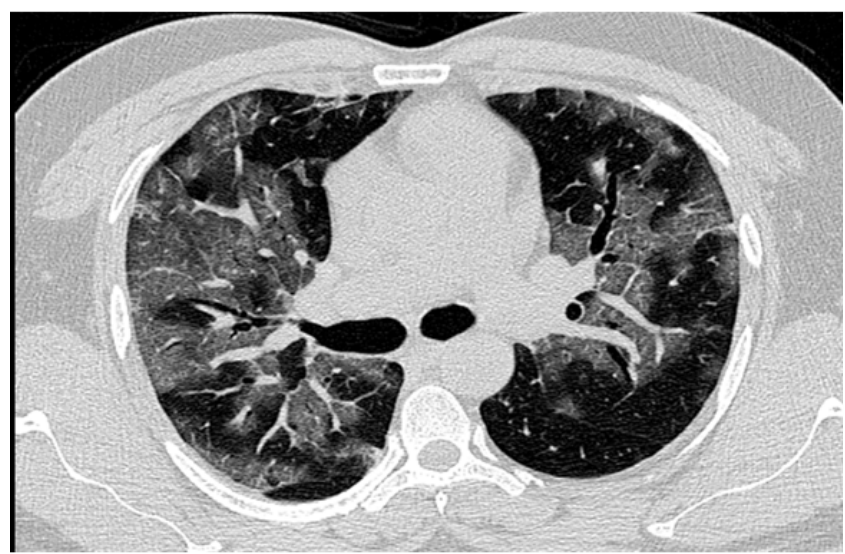

Fig. 1A. An axial section of the high resolution computed tomography chest image in a lung window showing patchy diffuse ground glass density s/o acute interstitial pneumonia.

to breathe spontaneously, sedation was ceased and his ventilator settings changed to a pressure support mode, with pressure support of $15 \mathrm{~cm}$ of $\mathrm{H} 2 \mathrm{O}$ and PEEP of $5 \mathrm{~cm}$ keeping the FIO2 at 40\%. This "spontaneous breathing trial" was planned to continue for forty-five minutes. However, within just a few minutes of such a trial, the patient immediately become tachypneic and tachycardic.

In keep with the hospital protocol, a patient meets the criteria of being ready to wean when he is hemodynamically stable and the primary disease process is resolving and good oxygen saturation is maintained. At this point, the ventilator settings are reduced and the patient is allowed to breathe on a spontaneous breathing mode on the ventilator. This patient appeared to meet all these criteria but could not pass a spontaneous breathing trial to meet the required extubating criteria.

Therefore subsequent to the failure of the "spontaneous breathing trial" reported above, his airway was secured immediately and ventilation was continued with a pressure control volume guarantee mode with volume of $400 \mathrm{ml}$ and a $100 \%$ fraction of inspired oxygen. Peak pressures reached to about $45 \mathrm{~cm}$ of water but oxygen saturations greater than $94 \%$ were maintained.

Clinically, there was a suspicion of pneumomediastinum, so rather than having a chest X-ray normally undertaken after intubation to confirm the cause of respiratory distress and rule out pneumothorax, an emergency high-dose contrast-enhanced computed tomography (CECT) was carried out. This showed an extensive mediastinal emphysema and pneumomediastinum. (Fig. 1B, C) 


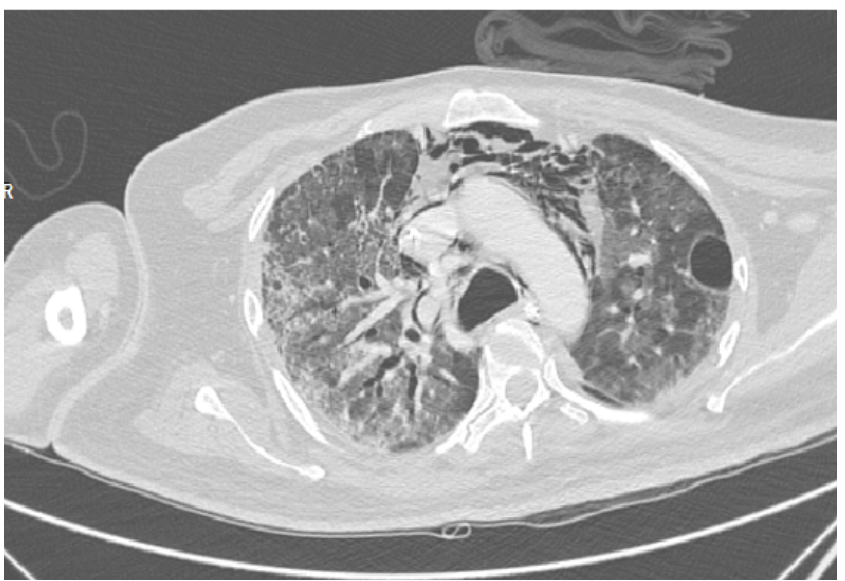

Fig. 1B. An axial section of the high resolution computed tomography chest image in a lung window showing Mediastinal emphysema (pneumomediastinum)

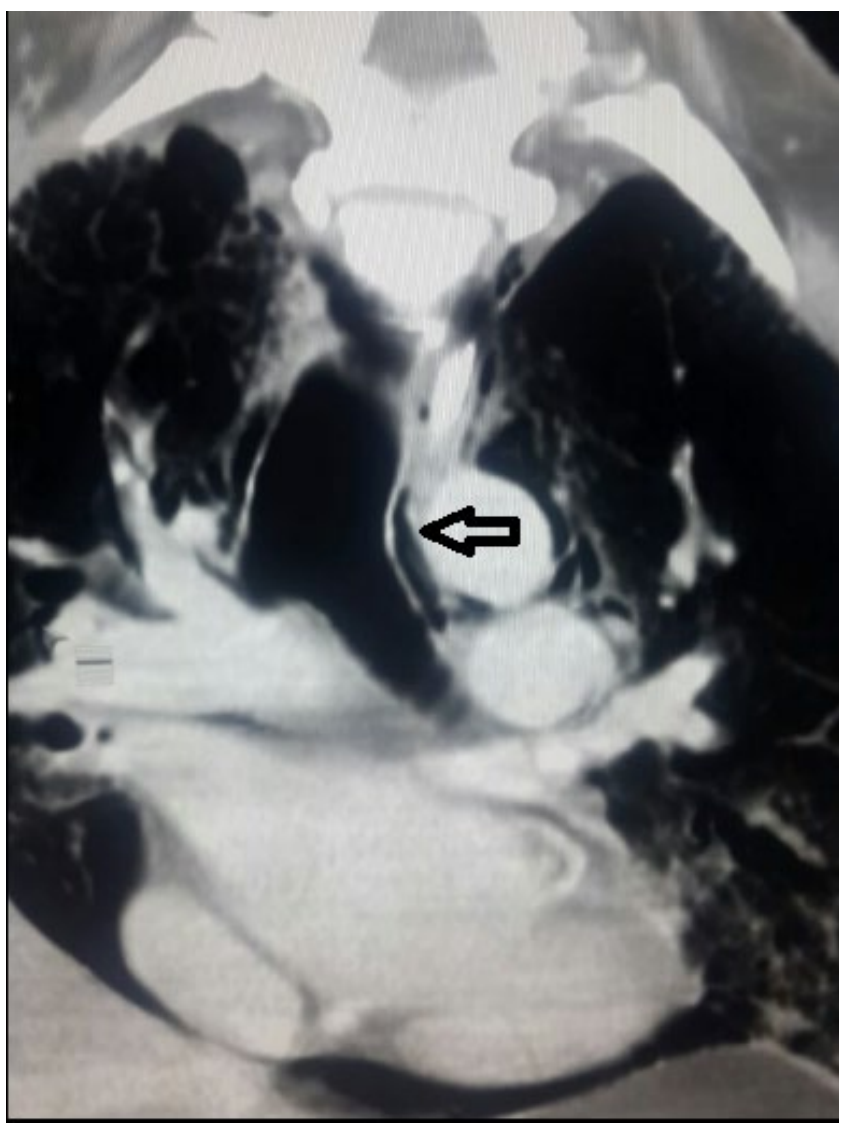

Fig. 1C. A coronal plane CT thorax showing extensive pneumomediastinum with peribronchial air (arrow) demonstrating the Macklin effect. Also we can see the continuous diaphragmatic sign.

Investigations of the retroperitoneum, esophagus and bronchi, to determine the possible sources of mediastinal air, were found to be normal.

Whenever, sedation was stopped, and a spontaneous breathing trial started, the patient would immediately commence coughing, become tachypneic and tachycardic. Concomitant with these coughing bouts, his subcutaneous emphysema would worsen.

This patient met the criteria of prolonged wean which according to the published consensus is seen in $<15 \%$ of intubated patients. One approach to manage a prolonged wean is tracheostomy, usually a bedside procedure done by the intensivist. He failed to respond to the gradual process of decreased ventilator support (weaning) over a one week period and consequently a decision was made to undertake a period of prolonged weaning and a surgical tracheostomy was performed. However in this case because of his extensive subcutaneous emphysema he was operated on by ENT surgeons, who performed a surgical tracheostomy.

Weaning problems persisted after tracheostomy. The patient was psychologically motivated and ready for weaning but when a spontaneous breathing trial was performed, even with a high pressure support of 16/8 and continuous positive airway pressure mode, he would fail within thirty minutes. Bilateral intercostal drains (ICD's) were inserted and a planned mediastinal drain was take into consideration, in the possible event there would be no improvement.

\section{RESULTS}

The hospital cardiothoracic team were requested to insert 30 French sized intercostal drains on both sides in the 4th intercostal space. After their insertion the drains were kept under a water seal of $2 \mathrm{~cm}$, and a successful respiratory wean planned to be performed within one week. (Fig. 2 A, B)

After an observation period of forty-eight hours he was moved to a step down ICU and then to a high dependency unit where the tracheostomy tube was removed and he was discharged. At that time he was clinically stable, able to walk around and perform his daily activities.

Since the recovery process of any injured tissue takes 6-8 weeks he was discharged with the bilateral intercostal drains in situ and nursing care was provided at home.

He was on follow up every week at the TCU clinic and after ten weeks of monitoring, the bilateral intercostal drains were removed by the resident duty doctor. 


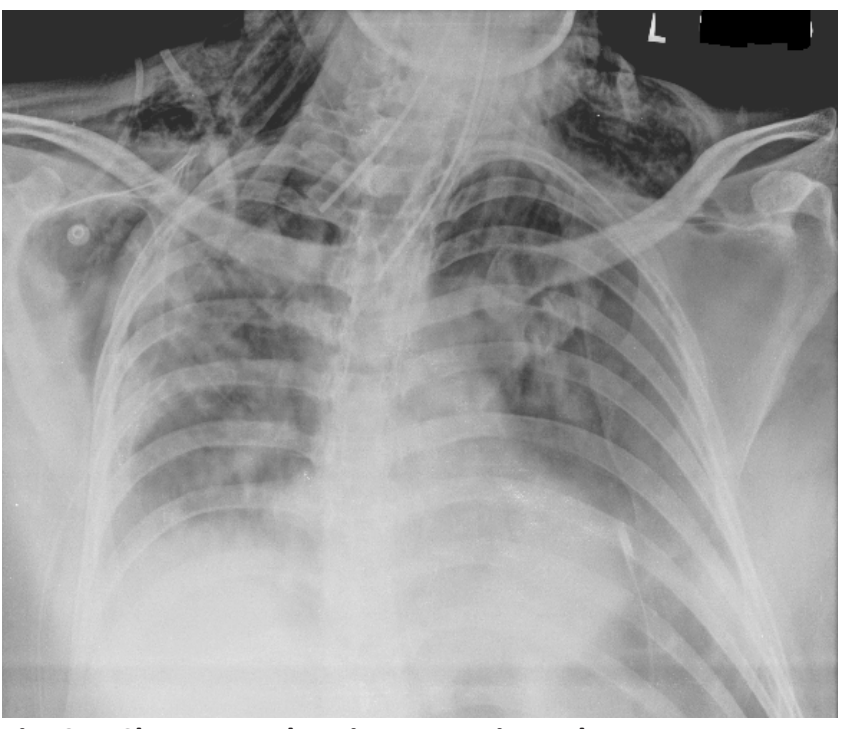

Fig. 2A. Chest xray showing extensive subcutaneous emphysema under both shoulders and pneumomediastinum.

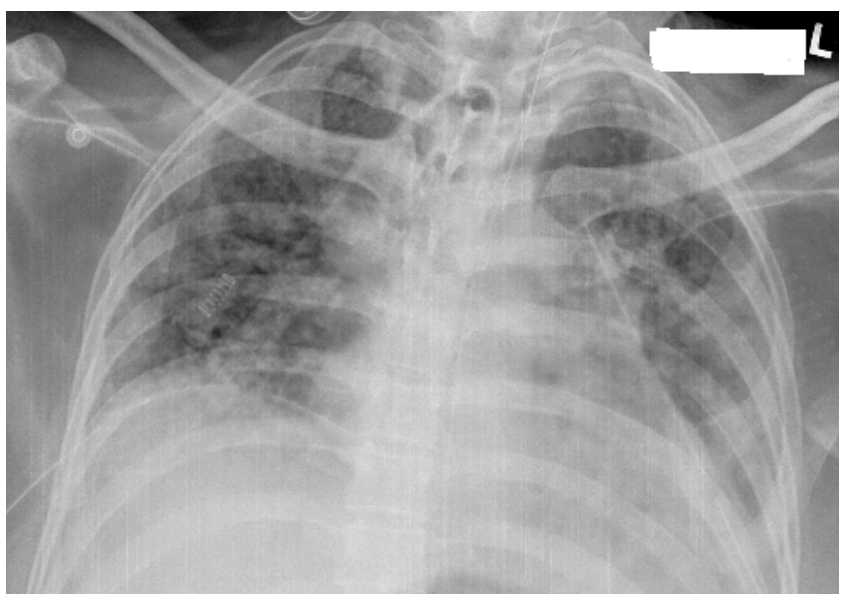

Fig. 2B. Complete resolution of above changes post bilateral intercostal drains.

\section{DISCUSSION}

Treatment management was based on guidance from literature reports of primary recurrent spontaneous pneumomediastinum. Although the patient did not have primary spontaneous pneumomediastinum, he had a similar condition arising as a complication of viral pneumonia. Pneumomediastinum is classified either as primary spontaneous or secondary [1]. Primary recurrent pneumomediastinum is an uncommon condition and its mechanism has been a matter of discussion. The typical patient is a young, otherwise healthy male, presenting with severe retrosternal chest pain and subcutaneous emphysema [2]. Though it is a benign condition, it often leads to unnecessary investigations, swallowing studies, dietary restriction, antibiotics and a long hospital stay [2]. Recurrent spontane- ous pneumomediastinum was described by Hamman (1939) when it was defined as air in the mediastinum with no apparent cause [3]. Risk factors included bronchial asthma, tobacco or drug use [4].

A commonly accepted mechanism is termed the Macklin phenomenon which suggests that coughing increases intra-alveolar pressure, causing alveolar rupture subsequent to which air tracks along the peribronchial and perivascular sheaths due to a pressure gradient between the mediastinum and subcutaneous tissue [5]. Alveolar rupture is usually precipitated by excessive coughing or the Valsalva manoeuvre due to barotrauma [6].

Clinical symptoms include severe retrosternal chest pain, subcutaneous emphysema, Hamman's signs of a crunching, rasping sound, synchronous with the heartbeat, and dyspnea [7]. Diagnosis is made mainly subsequent to a chest X-ray or CT. Despite the benign nature of the condition, an extensive workup is often undertaken to exclude hollow viscus perforation, though it is not usually required.

Several chest X-ray signs have been described and include subcutaneous emphysema, pneumo-pericardium, air around the pulmonary arteries, bronchi or aorta and continuous diaphragm sign [8]. Computed tomography (CT) has a high sensitivity and specificity and also helps rule out associated small pneumothoraces [9]. Management is mostly bed rest, observation, optimum sedation and antitussive agents like dextromethorphan [9]. Patients usually have a prolonged stay in the hospital but are usually discharged healthy.

The current patient was considered to have had a spontaneous pneumomediastinum since no other source of mediastinal air could be determined. Although it was a complication of $\mathrm{H} 1 \mathrm{~N} 1$ pneumonia, it was judged that Macklin's phenomenon was related to this case.

Malignant pneumomediastinum is a devastating complication, occurring when mediastinal air causes hypotension or difficulties in ventilation [10]. The ideal treatment is to insert a mediastinal drain with or without intercostal drainage [11]. In this case the patient did not have a tension pneumomediastinum, which, if present would have necessitated an emergency mediastinoscopy and mediastinal tube insertion [12].

The role of non-invasive ventilation (NIV) of patients in the treatment of acute respiratory distress syndrome (ARDS) has been investigated in several trials, the most 
significant of which is that of Bellani et al (2016) [13]. They concluded that although NIV decreased several complications associated with intubation it was linked to increased mortality especially in patients with a $\mathrm{PaO}_{2} / \mathrm{FiO}_{2}$ ratio $<150$. The patient that was the subject of this report had a $\mathrm{PaO}_{2} / \mathrm{FiO}_{2}$ ratio $>200$, which is classified as mild ARDS according to the Berlin definition of ARDS. Hence a decision was taken to continue with non-invasive respiratory support.

While on NIV support, patients may take small breaks to drink water or if they feel suffocated, the mask is removed for a short period. During one such NIV break, when a nurse had disconnected the mask to provide oral care, the patient developed a bout of excessive coughing and respiratory distress with tachypnea, and the need to use accessory respiratory muscles, accompanied by a fall in blood oxygen saturation falling below $80 \%$.

After waiting for over twenty days to wean him without success, a different treatment strategy was made for this patient based on the theory of Macklin's phenomenon. A thorough review of available published reports and expert advice was taken. A similar case was reported by Meireles (2011) which was managed conservatively [14], and the treatment of progressive pneumomediastinum by chest drain was reported by Holmes (1990) [15].

Because the patient was an Indian businessman involved in the textile industry, consideration was given to the possible presence of an occupational lung disease, such as "byssinosis" due to cotton dust exposure leading to impaired lung function. This was ruled unlikely as his medical history showed that, prior to the present episode, the patient had had no previous symptoms of breathlessness or wheezing suggestive of a chronic lung condition and CT scans of his thorax showed no chronic parenchymal process. A pulmonary function tests was also ruled out due to the patient's poor condition at that time [16].

It was hypothesized that by placing bilateral intercostal drains, in spite of the absence of a pneumothorax, a decrease the trans-pulmonary pressure gradient would be achieved which then in turn would reduce the intra-alveolar pressure and decrease the alveolar rupture. This would lead to a decrease in the pneumomediastinum and facilitate weaning. The possibility of placing a mediastinal drain was kept as an option, if further deterioration occured. This strategy was adopted in the knowledge that chest drains are simple to place and have far fewer complications than mediastinal drains.

The patient was discharged in a stable medical condition. He had mild pain at the intercostal drain sites measured on a visual analogue scale as $3-4 / 10$. He also had a slight occasional cough in spite of anti-cough medications. However this is expected in a healing patient and it did not affect his breathing. He was followed up at the outpatient clinic of the TCU who updated the ICU team regularly on his progress. He also personally came to visit the ICU team to extend his gratitude. At that time, a final assessment was made and an informed written consent from for publication was signed.

\section{- CONCLUSION}

This is a report of a case of spontaneous pneumomediastinum following a common viral illness. It is hoped that this report will provide guidance in the treatment of pneumomediastinum associated with $\mathrm{H} 1 \mathrm{~N} 1$ pneumonia.

\section{CONFLICT OF INTEREST}

None to declare

\section{REFERENCES}

1. Kouritas VK, Papagiannopoulos K, Lazaridis G, et al. Pneumomediastinum. J Thorac Dis. 2015;7(Suppl 1):S44-9.

2. Al-Mufarrej F, Badar J, Gharagozloo F, Tempesta B, Strother E, Margolis M. Spontaneous pneumomediastinum: diagnostic and therapeutic interventions. J Cardiothorac Surg. 2008;3:59.

3. Hamman L. Spontaneous mediastinal emphysema. Bull Johns Hopkins Hosp. 1939;64:1-21.

4. Caceres M, Ali SZ, Braud R. Spontaneous pneumomediastinum: a comparative study and review of the literature. Ann Thorac Surg. 2008;86:962-6.

5. Macklin MT, Macklin CC. Malignant interstitial emphysema of the lungs and mediastinum as an important occult complication in many respiratory diseases and other conditions: an interpretation of the clinical literature in the light of laboratory experiment. Medicine. 1944;23:281-358.

6. Bakhos CT, Pupovac SS, Ata A, Fantauzzi JP, Fabian T. Spontaneous Pneumomediastinum: An Extensive Workup Is Not Required. J Am Coll Surg. 2014;219(4):713-7.

7. Takada K, Matsumoto S, Hiramatsu T, et al. Management of spontaneous pneumomediastinum based on clinical experience of 25 cases. Respir Med. 2008;102(9):1329-34. 
Available online at: www.jccm.ro

8. Bejvan SM, Godwin JD. Pneumomediastinum: old signs and new signs. AJR Am J Roentgenol. 1996;166(5):1041-8.

9. Zylak MC, Standen RJ, Barnes RG. Pneumomediastinum revisited. Radiographics. 2000;20(4):1043-57.

10. Rajan A, Shrikhande D, Arora A, Manish K. Spontaneous Pneumomediastinum Complicating Pneumonia in Children. Med J Armed Forces India. 2010;66(1):83-5.

11. Tezel C, Varer P, Baysungur V, Okur E, Halezeroglu S. Spontaneous pneumomediastinum: report of two cases. Ulus Travma Acil Cerrahi Derg. 2011;17(4):368-70 .

12. Mohamed ISI, Lee Y-H, Yamout SZ, Fakir S, Reynolds AM. Ultrasound guided percutaneous relief of tension pneumomediastinum in a 1-day-old newborn Arch Dis Child
The Journal of Critical Care Medicine 2019;(5)1 • 33 Fetal Neonatal Ed. 2007;92:F458.

13. Bellani G, Laffey JG, Pham T, et al. Noninvasive Ventilation of Patients with Acute Respiratory Distress Syndrome. Insights from the LUNG SAFE Study. Am J Respir Crit Care Med. 2016;195(1):67-77.

14. Meireles J, Neves S, Castro A, França M. Spontaneous pneumomediastinum revisited, Respir Med. 2011;4(4):181-3.

15. Holmes KD, McGuirt WF. Spontaneous pneumomediastinum : evaluation and treatment. J Fam Pract. 1990;31(4):422-29.

16. Ali NA, Nafees AA, Fatmi Z, Azam SI. Dose-response of Cotton Dust Exposure with Lung Function among Textile Workers: MultiTex Study in Karachi, Pakistan. Int J Occup Environ Med. 2018;9(3):120-128. 